\title{
Indications for surgery in leprosy
}

\author{
MARCOS VIRMOND \\ Instituto Lauro de Souza Lima, Bauru, Spain
}

\section{Introduction}

What does surgery offer?

1. Correction of paralytic deformity by reconstructive surgery.

2. Correction of primary deformity by plastic surgery.

3. Prevention of worsening of deformity.

4. Prevention of deformity by nerve decompression.

5. Early healing of plantar ulcers.

Common sites of nerve damage and deformity

1. Ulnar nerve at elbow - clawed fingers and thumb.

2. Median nerve at wrist - fallen back or 'ape' thumb.

3. Lateral popliteal nerve at fibular neck - drop-foot.

4. Posterior tibial nerve in tarsal tunnel - clawed toes.

5. Facial nerve at zygomatic arch - lagophthalmos.

Common reasons for referring to a surgeon

1. Paralytic motor deformities of hand, foot and eyelids.

2. 'Neuritis' of a trunk nerve not improving after 2 weeks of medical treatment.

3. Primary deformities of nose, face and eyebrows.

4. Non-healing plantar ulcers.

Common symptoms

1. Trunk nerve enlargement and tenderness.

2. Pain in the nerve, at rest and on stretch.

3. Reduction or loss of sense of touch, pain and temperature.

4. Loss of sweating.

5. Motor weakness and increasing paralysis.

6. Nerve abscess.

\section{Rationale for surgery}

Leprosy affects nerves, skin and other soft tissues. Delay in treatment allows development of deformities indirectly by nerve damage (paralytic deformities and sensory loss) and directly 
by tissue damage (primary deformities). All these deformities can be corrected by surgery while sometimes nerve damage can be reversed or further damage prevented by nerve surgery. Surgery at any stage can stop further worsening of a deformity. Surgery gives best results when performed early, before secondary changes develop because of the abnormal structure. However, surgery is available only at special centres, so a proper referral process is essential.

\section{How are deformities caused?}

Nerve trunk damage produces deformities indirectly through sensory loss and loss of motor power. Loss of pain and other sensation increases the vulnerability to injury and infections, and results in ulcers in the palm and sole. Loss of motor power causes muscular imbalance leading to deformity. For example, loss of flexor power at the metacarpophalangeal (MP) finger joint causes hyperextension of the MP joints and flexion of distal joints- the clawing deformity. The disability here is of grasp, because the fingers now roll over instead of flexing first at the MP joints. Secondary to these deformities, because of altered position and dynamics, there occur other complications like joint contractures, adaptive shortening of flexors, ulceration at knuckles and under the metatarsal heads and finger tip flexion.

The primary deformities, like depressed nose and loss of eyebrows, are seen mainly when treatment is delayed in lepromatous patients. These can be well corrected by plastic surgery.

\section{Common reasons for referral to a surgeon}

There are a group of conditions in leprosy patients that cannot be adequately managed by basic health personnel and should be referred to a surgeon. The main conditions are listed below.

\section{PARALYTIC DEFORMITIES AND DISABILITIES OF HANDS}

Definite clawing of the fingers and lack of opposition of the thumb that cannot be restored by physiotherapy. This condition requires tendon transfer procedures. Surgery can improve clawing of the fingers, allowing patients to grasp large objects and improving pinch with the non-paralysed thumb, thus restoring adequate hand function to daily activities and labour.

\section{PARALYTIC DEFORMITIES AND DISABILITIES OF FEET}

Definite droop foot and clawed toes are conditions which health education and prevention of disabilities can prevent from worsening, but only surgery can restore dorsiflexion of the foot and reverse clawing of the toes. Tendon transfers are the procedure of choice to restore dorsiflexion to a dropped foot, with the aim of restoring the normal gait, thus preventing destructive ulcers in the lateral border and fixed deformity of the ankle with equinus-varus. Tendon transfers can also restore, to some extent, a straight position of the toes, preventing ulcers in the dorsum. Correction of the clawed toes leads also to an improved distribution of pressure under the metatarsal heads, so preventing plantar ulcers in anaesthetic feet. 


\section{LAGOPHTHALMOS}

Failure to close the eyes is a serious and threatening condition. Exposure keratitis due to drying of the cornea can lead to corneal ulceration and, ultimately, to blindness. This condition can be treated, for a while, by oil drops and corrective lenses. Surgery can offer a more definite protection of the exposed eye. Temporal muscle transfer, tarsal sling and tarsorraphy are procedures that can provide an adequate and long standing protection to the cornea of an eye which cannot be closed due to paralysis of the orbicularis muscle.

\section{ULCERS AND INFECTIONS}

Although plantar ulcers are commonly treated by dressings in ambulatory patients, there are some situations that call for surgical removal of dead bone and other tissues. A constantly discharging ulcer that does not heal despite adequate cleaning and dressings is a clear sign that some necrotic bone or infected tissue (tendon or capsule) is preventing ulcer healing and requires surgery. Deep spaces, synovial sheath and pulp infection in the hand are common situations that can go unnoticed due to loss of sensation and consequent absence to pain. These infections should be identified early and promptly treated by adequate surgical drainage to prevent its progression, which eventually leads to shortening of the fingers and severe skin contractures, i.e. a non-functional hand.

\section{Neuritis}

The trunk nerves in leprosy are involved at particular sites, where the nerve passes through a fibro-osseous tunnel. Here, the nerve is compressed when enlarged. The symptoms are due to inflammation and compression, pain, tenderness, stretch pain on flexion of the joint and sensorimotor and autonomic dysfunction distally. Early diagnosis and treatment can reverse the nerve damage. Sometimes, surgical decompression is required.

\section{COMMON SYMPTOMS}

\section{Paralytic deformities of the hand}

Ulnar nerve paralysis:

1. Moderate abduction of the little finger.

2. Wasting of the hypothenar eminence and of the first web.

3. Inability to spread fingers.

4. Clawing of fingers, especially ring and little fingers.

5. Difficulty in grasping.

6. Sensory loss in little finger and half of ring finger.

7. Flexion of thumb tip during pinch.

Median nerve paralysis:

1. Progressive weakness of thumb opposition.

2. Wasting of thenar region.

3. Sensory loss in lateral two-thirds of hand.

Ulnar-median paralysis (commoner than median paralysis alone):

1. Clawing of all the fingers. 
2. Loss of opposition.

3. Only key pinch is available, normal grasp and pinch are not available.

4. Sensory loss in the palmar aspect of the whole hand.

\section{Paralytic deformities of the leg and foot}

Lateral popliteal nerve paralysis:

1. Wasting of anterolateral compartment of the leg.

2. Inability to dorsiflex the ankle and toes.

Posterior tibial nerve:

1. Wasting of the foot.

2. Inability to squeeze the toes together or to spread them.

3. Inability to keep the toes straight while elevating the heel.

4. Sensory loss in the sole (plantar aspect).

\section{Facial nerve}

1. Inability to close the eyelids.

2. Dry eyes.

3. Sometimes corneal anaesthesia.

\section{Prerequisites for surgery of primary deformities}

1. Adequate antileprosy treatment.

2. Bacterial index preferably 2 or less.

3. Adequate control of local infection.

The symptoms of paralytic deformities depend on the nerve involved and its distal distribution.

In the hand, ulnar nerve paralysis is the commonest, followed by the combined ulnarmedian paralysis. In ulnar nerve paralysis (Figure 1), the medial one-third of the palm has sensory loss, while early mild abduction of the little finger is due to paralysis of the lumbrical and interossei and excessive action of the extensor tendon, which has a slight ulnarwards insertion of this finger (Wartberg's sign). With progression of nerve damage, there is now increasing clawing of the fingers with MP hyperextension and flexion of the distal joints, because of unbalanced action of the extensors. All the interossei, which provide the normal flexor force at the MP joint, are paralysed here.

Isolated median nerve paralysis is uncommon. Usually, there is a combined ulnarmedian paralysis (Figure 2), with clawing of all the fingers and loss of thumb opposition, which is a very disabling condition, as the patient cannot pick up objects. The whole palm of the hand also has sensory loss. This is a hand with hardly any function.

In lateral popliteal nerve paralysis, there is loss of function of the dorsiflexors. The result is inability to dorsiflex the foot and toes (drop foot). The gait is now with the forefoot striking the ground first - the high stepping gait. Often the evertors, the peronei muscles, are also paralysed, producing inversion deformity and later ulceration on the lateral border of the foot because of excessive pressure during walking and standing. Long-standing paralysis of these 

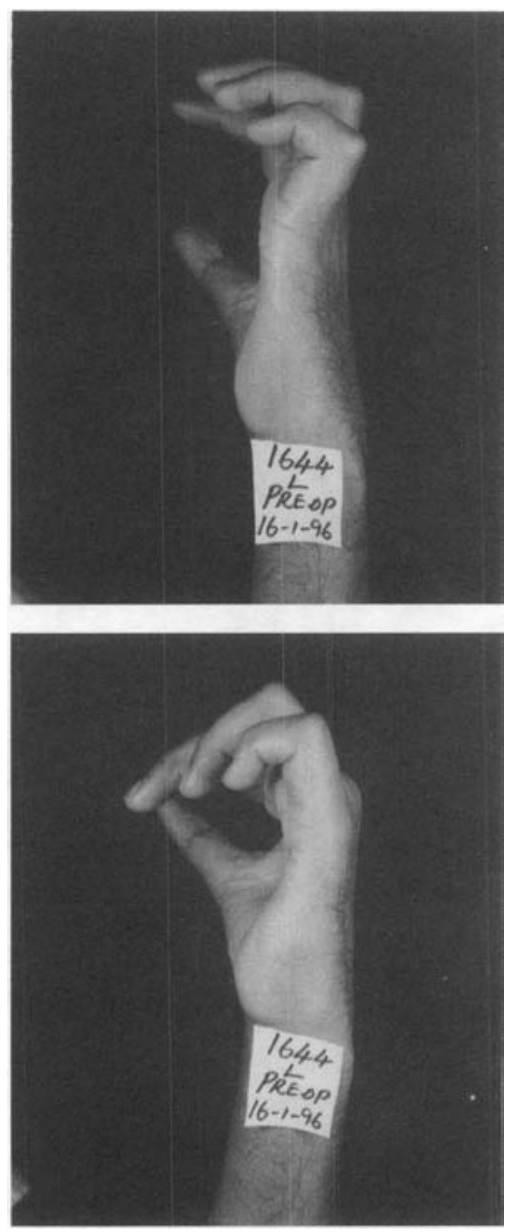

Figure 1. (a) Wasting of abductor digiti minimi, the lumbricals and interossei. (b) Clawing of the fingers with MP hyperextension and flexion of the distal joints.

muscles leads to rigid equinus varus deformity - the foot is fixed in plantar flexion with internal deviation.

In posterior tibial paralysis (Figure 3), the intrinsic muscles of the foot are paralysed, causing progressive hyperextension of metatarsophalangeal joints and flexion of the distal phalanxes (clawed toes). The condition of clawed toes has the same pathophysiology as clawed hand. The dorsum of the toes is exposed to excessive pressure as well, as the toe tip bends downward and is flattened because the toe pad is bearing at normal pressure. The toe tip is not anatomically designed to support pressure in the way that the toe pad is. With progression of the deformity, the metatarsal heads are overexposed in the plantar surface of the forefoot. Increased weight bearing in this area produces plantar ulcer. This is why clawed toes in an anaesthetic foot produce plantar ulcers in the forefoot.

In facial nerve paralysis, the eyelids are unable to close fully, the eye remains open, and becomes dry. Exposure keratitis may occur and lead ultimately to blindness. Thus this 


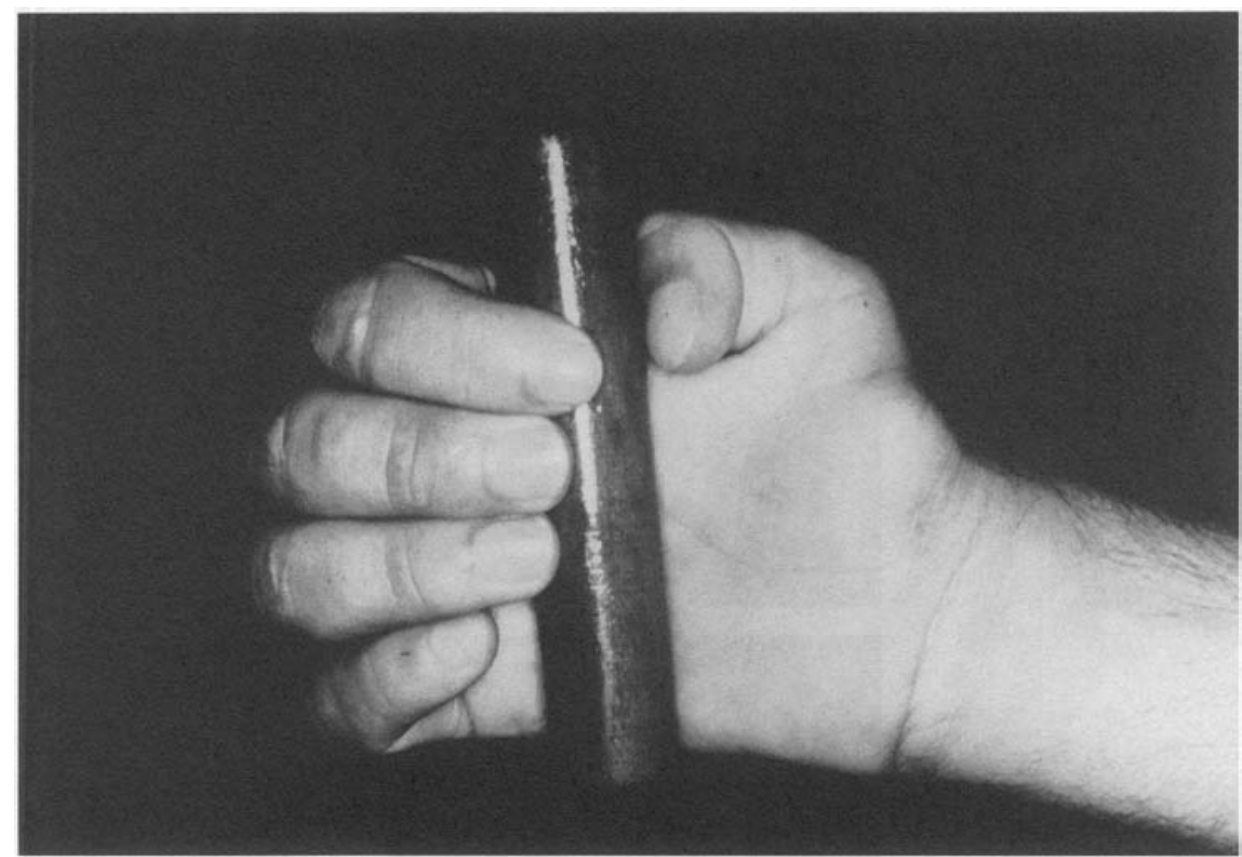

Figure 2. Loss of group in combined ulnar/median paralysis (courtesy of F. Duerkson).

condition needs to be treated urgently to prevent damage to the eye. Early medical and physical therapy treatment is often successful, otherwise surgery is essential to save the eye.

\section{Evaluation and monitoring}

This is done using graded nylon filaments for testing sensation. Motor power is tested by using the original or modified MRC scale. All clinical signs are also graded. Repeated monitoring is essential to observe progress, or otherwise, and take preventive action in time.

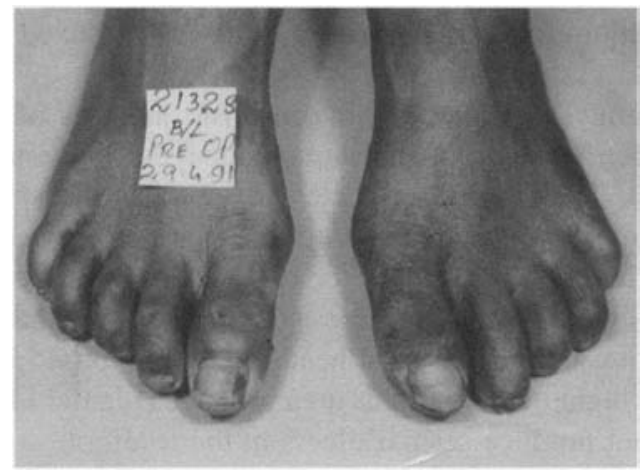

Figure 3. Posterior tibial nerve paralysis causing clawed toes (courtesy of D. Palande). 


\section{Indications for surgery}

Nerve surgery

1. Inadequate improvement after 2 weeks of medical treatment.

2. Chronic nerve pain.

3. Nerve abscess.

4. Recurrent neuritis.

5. Ischaemia plantar ulcers or ulcers which are not healing.

Established paralytic deformities

1. One year duration of paralysis.

2. Clawing of fingers, with or without clawing of the thumb.

3. Foot drop of 6 months or longer duration.

4. Clawed toes.

5. Lagophthalmos of longer than 1 month duration.

Ulcer surgery

1. Non-healing ulcers.

2. Ulcers with septic or synovial discharge.

3. Ulcers with boot involvement.

Primary deformities

Any primary deformity, once it has occurred, is irreversible and the patient should be referred for surgical evaluation to decide when surgery should be done.

Palatal and nasal deformities occur in neglected cases of lepromatous leprosy. Early prevention is by nasal care during treatment. Plastic surgery procedures can correct these deformities.

Facial wrinkles and sagging ear lobes result from subcutaneous infiltration and are easily corrected by face lift and plastic surgery.

Gynaecomastia occurs secondary to hormonal changes following testicular atrophy and treatment is surgical.

There is a group of uncommon conditions that can be corrected by surgery for functional and cosmetic reasons. Wrist drop alone is rarer than a triple nerve paralysis, which is a very disabling condition needing multiple tendon transfers for its correction. Club foot needs radical correction and fusion of joints. Sagging ear lobes, loss of eyebrows and gynaecomastia all need correction for psychological reasons. Nasal and palatal deformities are very disabling and corrective plastic surgery is essential. The results of surgery in all these conditions are excellent.

\section{Prerequisites for surgery}

In all cases, before surgery is done, the paralysis should be of 1 year duration, i.e. irreversible, and the patient should be receiving multi-dose therapy and without recent reactions. The bacterial index of skin smears should be $\leq 2$. For primary deformities like those of the nose or palatal performation, the disease must be inactive. In any surgery, there must not be any septic focus anywhere, as sepsis spoils the result completely. 
Any established deformity is an indication, and the earlier the surgery is performed, the better is the result. For nerve surgery, the main indications are failure of medical and physical therapy, recurrent neuritis, intractable pain and a nerve abscess. For the posterior tibial nerve, decompression is not only of the nerve, but also of the blood vessels, and this improves the blood supply.

\section{Ulcer surgery}

Surgery is required here mainly to remove a septic focus, open up any tracks, and to remove any dead bone. Sometimes, in cases of recurrent ulceration of the forefoot, metatarsectomy is needed, while for a heel ulcer, some flap procedures may be required. The mainstay for prevention and treatment of ulcer recurrence is care of the foot and proper orthotic devices, including footwear.

\section{Conclusion}

Surgical treatment is essential to comprehensive care of the leprosy patient. Indications are clearly defined and should be known to all health personnel involved in leprosy. If surgical facilities are not available locally, an efficient referral system needs to be established.

\section{Further reading}

Duerksen F, Virmond M. Cirurgia Reparadora e Reabilitracao em Hanseniase. Palavra e Acao-TALMILEP, Rio de Janeiro, 1997.

Fritschi EP. Surgical reconstruction and rehabilitation in le prosy. Director for Southern Asia, Leprosy Mission, New Delhi, 1992.

McDowell F, Enna C. Surgical rehabilitation in leprosy. Williams \& Wilkins, Baltimore, 1974.

Srinivasan H, Palande DD. Essential surgery in leprosy - techniques for district hospitals. World Health Organization, 1997. 